\title{
New Antibacterial Agents must, but Easy-to-Implement Assays First
}

\section{Boobalan Pachaiyappan* \\ Department of Chemistry, Northwestern University, 2145 Sheridan Road, Evanston, IL 60208-3113, USA}

*Corresponding author: Boobalan Pachaiyappan, Department of Chemistry, Northwestern University, 2145 Sheridan Road, Evanston, IL 60208-3113, United States, Tel: +1 847-491-3741; E-mail: boobalanp@gmail.com

Rec date: June 17, 2015; Acc Date: June 19, 2015; Pub date: June 22, 2015

Copyright: $\odot 2015$ Pachaiyappan B. This is an open-access article distributed under the terms of the Creative Commons Attribution License, which permits unrestricted use, distribution, and reproduction in any medium, provided the original author and source are credited.

\begin{abstract}
Therapeutic validation of peptidyl-tRNA hydrolase (Pth) inhibition to discover novel antibacterial drug scaffolds is encumbered by lack of medicinal chemistry efforts and a simple easy-to-implement assay procedure for the analysis of Pth activity. In this issue, Holloway et al. have demonstrated that such a quantification assay is possible. This should speed-up inhibitor screening for identification of novel hits.
\end{abstract}

\section{Commentary}

Traditional antibiotics kill bacteria or inhibit its growth by blocking the synthesis of cell wall, proteins and nucleic acids. However, due to the emergence of multi-drug resistant bacteria, current antibiotics either as a stand-alone treatment, or in combination, have received only partial success. This scenario gets further attenuated by acquired resistance via additional mechanisms such as target mutations, increased expression levels of efflux pumps and antibiotic-degrading enzymes. Clearly drug discovery efforts that are aimed to design alternative molecular scaffolds to target antibacterial growth and acquired resistance is urgently required [1].

Inhibition of bacterial protein synthesis has been an active area of research for over several decades. Majority of antibiotics that block the protein synthesis (for example doxycycline and streptomycin) function by interfering with the ribosomes. The resistance faced by existing antibiotics and a decline in the development of novel antibiotics calls for an improved understanding of bacterial protein synthesis so that alternative therapeutic approaches can be envisioned. One of the untoward events that happen during bacterial protein synthesis is "ribosome stalling" - a process that involves sudden stopping of translation of genetic information from mRNA to proteins. Whereas several reasons contribute to this anomaly - for example, tRNA starvation, defective mRNA - bacteria has developed protective mechanisms to release the retention of immature peptidyl tRNA. One such mechanism happens through peptidyl-tRNA hydrolase (Pth), an esterase that cleaves the ester bond between the Cterminal end of the peptide and the 2'- or 3'- hydroxyl of the ribose end of tRNA (see Figure 1 for a simplified reaction) [2,3]. The recycling activity of $\mathrm{Pth}$ is considered critical for bacterial survival because any lack of it may lead to the accumulation of immature peptidyl tRNA that is toxic to cells [4]. There is a growing interest in Pth for the development of novel antibiotics considering that its inhibition may either impair the translation or slow down bacterial protein biosynthesis [5].

Despite the availability of Pth crystal structures in Protein Databank, medicinal-chemistry based efforts for the development of Pth inhibitions are scarce, partly because of a lack of an assay that overcomes current.

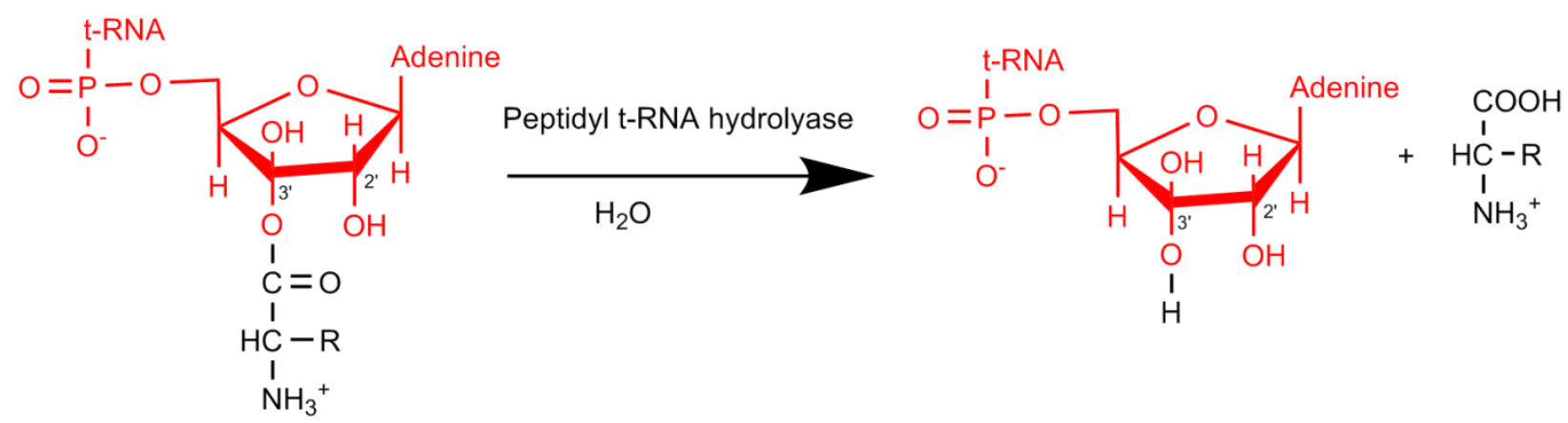

Figure 1: A simplified reaction of peptidyl-tRNA hydolyase.

limitations including use of radiolabeling agents, cost, number of steps involved and incompatibility with other Pth family of enzymes. In this issue, Holloway et al. have attempted to address this, at least in part, for monitoring the Pth hydrolysis of peptidyl-tRNA [6].

Their research started with the goal of developing a robust and expeditious assay method to analyze the Pth enzymes-initiated cleavage of peptidyl-tRNA substrate. This functional assay involved application of acid-urea gel electrophoresis. In the first step, the bacterial Pth enzymes were cloned, expressed in E. Coli and purified using a previously described procedure. The second step involved culturing of bacterial substrate (bulk peptidyl-tRNAs) in C600 pth (Ts) cells and a subsequent resuspension of the pellets in diethylpyrocarbonate (DEPC) treated double-distilled water $\left(\mathrm{ddH}_{2} \mathrm{O}\right)$. The third step involved adding necessary ingredients to initiate the cleavage reaction. In a typical cleavage reaction of $20 \mu$ total volume, 
the authors used $1 \mu \mathrm{l}$ Pth, $10 \mu \mathrm{l}$ reaction buffer, $7.5 \mu \mathrm{l}$ DEPC $\mathrm{ddH}_{2} \mathrm{O}$ and $1.5 \mu \mathrm{l}$ peptidyl-tRNA. During $\mathrm{IC}_{50}$ determination studies, $4 \mu \mathrm{l}$ of $\mathrm{H}_{2} \mathrm{O}$ is replaced by equal volumes of inhibitor solutions or solvent. The fourth step involved running single layer acid-urea gels for the separation of peptidyl-tRNAs using 5S RNA as an internal reference. The gels were ran at constant voltage until the bromophenol dye reached the bottom. A plot of migration distance versus intensity was constructed using Image and analyzed using a custom script developed using Mathematica. The overall procedure is illustrated as a simplified cartoon in Figure 2.

Even though the technique acid-urea gel electrophoresis is used routinely in molecular biology, this is the first time it has been elaborately carried out for studying the hydrolysis of Pth [7]. Also, the described method involved using a bulk, peptidyl-tRNA as a substrate compared to an expensive acylated analogue that has been used traditionally. It must be kept in mind that the former brings additional challenges such as inhomogeneity of the substrate. To achieve optimal separation, the authors have optimized various parameters including acrylamide gel percentage, running voltage and temperature of the running buffer. At the first sight, it might appear that the bands are not well separated, but it must be kept in mind that the substrate used is an inhomogeneous collection of heterogeneous tRNA and peptides, and hence a clear demarcation between bands are not always possible. In general, for biological samples, usually low temperature and voltage are preferred to prevent accidental damage of samples. Interestingly, in the present case, maintaining a running buffer temperature between $8-12{ }^{\circ} \mathrm{C}$ and a high running voltage of $110 \mathrm{~V}$ resulted in optimal gel performance and hence an improved separation.

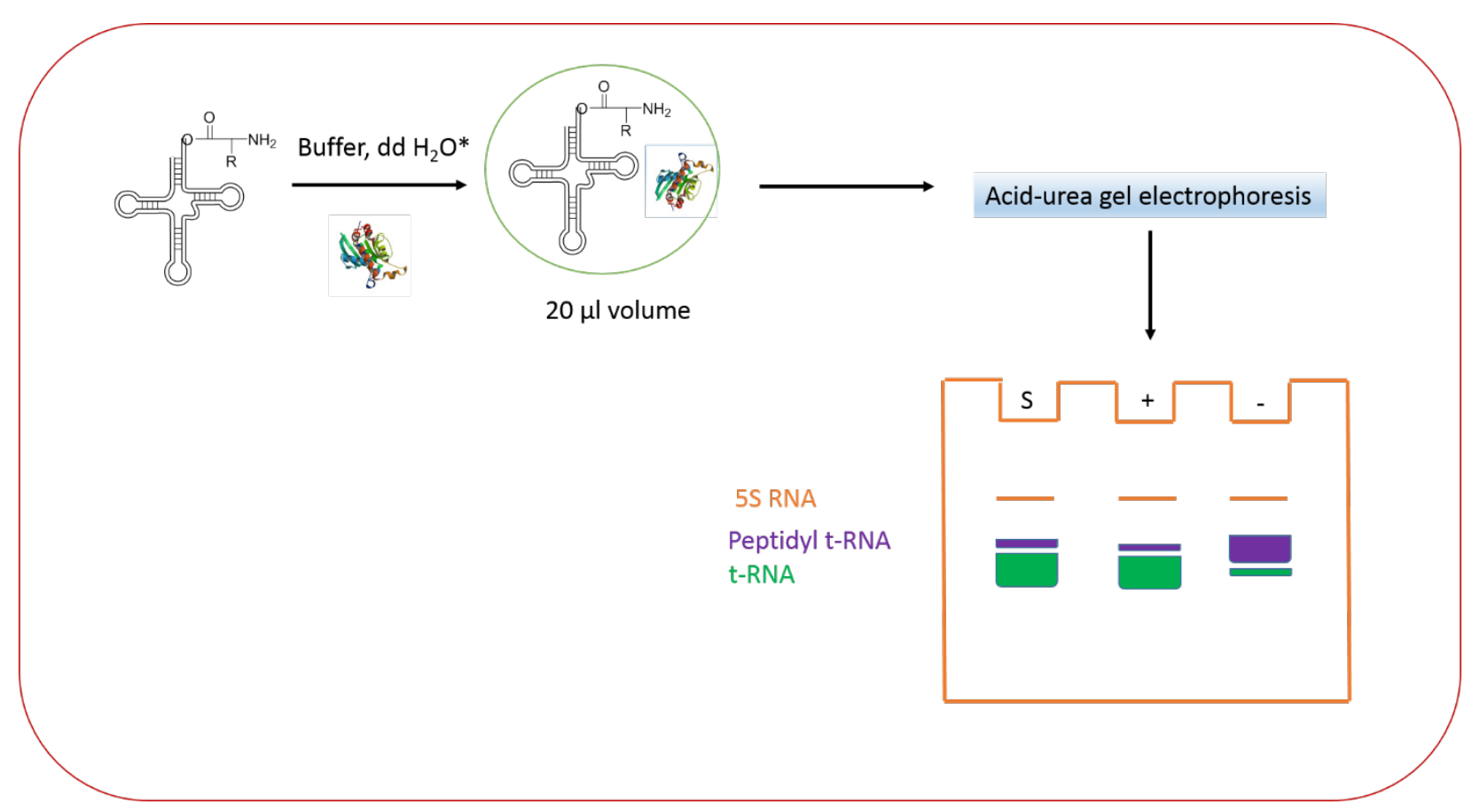

Figure 2: Acid-urea gel electrophoresis of Pth hydrolysis of peptidyl-tRNA. S - Sample, + positive (cleaved) control, - negative (uncleaved) control.

It is also shown here that the present method can be readily tweaked to measure Pth enzyme activity, to elucidate kinetic parameters and to screen chemical compounds to determine Pth inhibition. As an example, the authors presented screening results of crude natural product extracts against Gram-negative $P$. aeruginosa Pth1. Specifically, the natural product Aloe arborescens was measured to have an $\mathrm{IC}_{50}$ value of $72 \mu \mathrm{g} / \mathrm{ml}$. While this is not a great inhibition, subsequent structure-based optimization can be initiated to boost potency!

From a pharmaceutical standpoint, a bigger question that remains to be answered is how bacteria will react to small molecule-mediated Pth-inhibition. It has been well established that bacterial Pth enzymes are highly conserved and are structurally dissimilar to human counterparts. Therefore, small molecule-mediated Pth-inhibition should not only work for a wide array of Pth enzymes across the species but should also result in minimal/no side-effects. However, whether this might trigger a mutation in target active site or overproduction of target enzyme remains to be explored. In the case of conventional antibiotics, either mutation or target enzyme overproduction occurs over continued usage leading to a decline in antibiotic efficacy.

Cellular protein synthesis is a tightly regulated network that happens in ribosomes. In bacteria, various rescue factors such as tmRNA-SmpB, ArfA, ArfB, EF4, EF-P, RF1/3, Rf2/3 and Pth enzymes play a crucial role to prevent ribosome stalling [8]. Therefore, another interesting area of research is to determine whether Pth inhibition might activate 'Pth-independent' quality control mechanisms using rescue factors outlined above which might lead to prolonged bacterial survival.

The functional significance of other Pth enzymes (Pth2-4) have not been completely elucidated, but efforts were taken already. Once the biochemistry of Pth enzymes has been completely understood, a 
Citation: Pachaiyappan B (2015) New Antibacterial Agents must, but Easy-to-Implement Assays First. Biochem Anal Biochem 4: 190. doi: 10.4172/2161-1009.1000190

Page 3 of 3

structure-based screening can be carried out to identify novel small molecule hits that can be eventually optimized to a drug-like molecule. There is a huge innovation gap in antibacterial drug discovery over the last few decades, therefore the timing is perfect for continued Pth exploration. Computer modeling techniques will play a crucial role during the optimization of binding and property parameters [9].

In summary, the study by Holloway et al. provides a rapid, inexpensive method for the quantitative analysis of Pth hydrolysis activity. This makes it possible to explore the therapeutic implications of Pth1 in vitro with an ultimate aim to progress the antibiotic drug discovery.

\section{References}

1. Andersson DI, Hughes D (2010) Antibiotic resistance and its cost: is it possible to reverse resistance. Nature reviews Microbiology 8(4): 260-271.

2. Cuzin F, Kretchmer N, Greenberg RE, Hurwitz R, Chapeville F (1967) Enzymatic hydrolysis of N-substituted aminoacyl-tRNA. Proceedings of the National Academy of Sciences of the United States of America 58(5): 2079-2086.

3. Kossel H (1970) Purification and properties of peptidyl-tRNA hydrolase from Escherichia coli. Biochimica et biophysica acta 204(1): 191-202.

4. Das G, Varshney U (2006) Peptidyl-tRNA hydrolase and its critical role in protein biosynthesis. Microbiology 152: 2191-2195.

5. McFeeters RL (2013) Recent Antimicrobial Developments Targeting Peptidyl-tRNA Hydrolases. JSM Biotechnol Bioeng 1: 1006.

6. Holloway WB, McFeeters H, Powell AM, Nidadavolu GS, McFeeters RL (2015) A Highly Adaptable Method for Quantification of Peptidyl-tRNA Hydrolase Activity. Biochem Anal Biochem.

7. Kohrer C, Rajbhandary UL (2008) The many applications of acid urea polyacrylamide gel electrophoresis to studies of tRNAs and aminoacyltRNA synthetases. Methods 44(2): 129-138.

8. Giudice E, Gillet R (2013) The task force that rescues stalled ribosomes in bacteria. Trends in biochemical sciences 38(8): 403-411.

9. Kapetanovic IM (2008) Computer-aided drug discovery and development (CADDD): in silico-chemico-biological approach. Chemico-biological interactions 171(2): 165-176. 